\section{The efficacy and safety of the timolol/ dorzolamide fixed combination vs latanoprost in exfoliation glaucoma}

AGP Konstas ${ }^{1}$, VP Kozobolis², I Tersis' ${ }^{1}$ J Leech ${ }^{3}$ and WC Stewart ${ }^{3,4}$

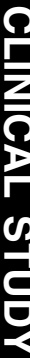

Abstract

Purpose To evaluate the safety and efficacy of the timolol/dorzolamide fixed combination $v$ s latanoprost $\mathbf{0 . 0 0 5 \%}$ in exfoliation glaucoma patients.

Methods We randomized in an observermasked fashion 65 newly diagnosed exfoliation glaucoma patients to either the timolol/dorzolamide twice daily or latanoprost daily treatment for 2 months and then crossed these over to the other treatment.

Results A total of fifty-four patients completed the study. After 2 months of chronic dosing, the morning intraocular pressure (IOP) (10:00) was reduced from a baseline of $31.2 \pm 6.5 \mathrm{mmHg}$ to $18.1 \pm 3.0$ with the fixed combination and to $18.9 \pm 4.1 \mathrm{mmHg}$ with latanoprost $(P=0.21)$. Six patients were discontinued early from both treatment periods owing to inadequate IOP control and two others were discontinued from latanoprost treatment only. The fixed combination showed a significantly greater incidence of taste perversion $(P<0.001)$ and stinging upon instillation $(P=0.036)$, while latanoprost showed a trend for increased conjunctival injection $(P=0.056)$. However, five patients demonstrated either bradycardia or asthmatic symptoms with initiation of the fixed combination therapy. One patient on latanoprost complained of dizziness. Patient preference was generally given to latanoprost (63 vs 20.3\%) mainly because of its once daily dosing $(P<0001)$.

Conclusions This study suggests that both latanoprost and the timolol/dorzolamide fixed combination are efficacious in the treatment of newly diagnosed exfoliation glaucoma.

Eye (2003) 17, 41-46. doi:10.1038/

sj.eye. 6700257

Keywords: exfoliation glaucoma; latanoprost; Cosopt; timolol; dorzolamide

Introduction

Exfoliation syndrome occurs worldwide and has recently been recognized to be the most common identifiable cause of open-angle glaucoma, ${ }^{1}$ accounting, in some countries, for the majority of the glaucoma. ${ }^{1,2}$ It has been estimated that the number of people with exfoliation in the world vary between 60 and 100 million, ${ }^{1}$ is increasing with ageing and, thus, the socioeconomic importance of exfoliation glaucoma has increased considerably in recent years. The response of exfoliation glaucoma to the new antiglaucoma medications may differ from that of primary open-angle glaucoma and thus it is important to evaluate the treatment response of this glaucoma and determine the most effective initial and stepwise treatment regime.

Latanoprost (Pharmacia, Peapack, NJ, USA) and the timolol maleate/dorzolamide fixed combination (Cosopt, Merck, Bluebell, PA, USA) both represent important formulations for the treatment of ocular hypertension and primary open-angle glaucoma. Recently, Fechtner $e t a l^{3}$ compared the daytime intraocular pressure (IOP) of latanoprost $v$ s the fixed combination product. They found a statistically similar IOP between the two products with a reduced diurnal IOP from untreated baseline of $26 \%$ for the fixed combination and $27 \%$ for latanoprost. ${ }^{3}$

More recently, Konstas et $a l^{4}$ evaluated whether latanoprost dosed in the evening would have a greater ocular hypotensive effect than the fixed combination for a 24-h diurnal curve. They showed almost similar daytime
${ }^{1}$ University Department of Ophthalmology AHEPA Hospital

Thessaloniki, Greece

${ }^{2}$ Department of Ophthalmology and Vardinoyannion Eye Institute of Crete

University of Crete

Greece

${ }^{3}$ Pharmaceutical Research Corporation

Charleston, SC, USA

${ }^{4}$ Carolina Eye Institute at the University of South Carolina School of Medicine Columbia, SC, USA

Correspondence: Dr AGP Konstas University Department of Ophthalmology AHEPA Hospital

1 Kyriakidi Str, Thessaloniki Greece

Tel: + 310994774

Fax: + 310245499

E-mail: konstas@

med.auth.gr

Received: 29 August 2001 Accepted in revised form: 8 March 2002

This study was not supported by an outside granting company or agency 
IOP, but at the 22:00 time point there was a greater IOP effect from the fixed combination of approximately $2.3 \mathrm{mmHg}$. ${ }^{4}$ This was consistent with this group's previous findings that latanoprost dosed in the evening provided a slightly less ocular hypotensive effect compared to morning dosing. ${ }^{5}$

The above studies were performed in patients with ocular hypertension or primary open-angle glaucoma. Patients with exfoliation syndrome have, on average, higher baseline IOP than those with primary open-angle glaucoma. ${ }^{1,2,6}$ The higher baseline pressures might allow for greater absolute decreases in the IOP with initial treatment, thus exaggerating any potential differences between the two medicines. ${ }^{7}$ The purpose of this study was to evaluate the safety and efficacy of the timolol/ dorzolamide fixed combination vs latanoprost in exfoliation glaucoma patients.

\section{Materials and methods}

\section{Patients}

Patients included in this study were newly diagnosed, previously untreated with antiglaucomatous medicines and were enrolled consecutively in two centres (University Departments of Ophthalmology in Thessaloniki and Crete, Greece). Patients were older than the age of 38 , had typical signs of exfoliation syndrome ${ }^{8}$ along with either elevated IOP or typical glaucomatous optic discs or visual field damage from glaucoma, and had an untreated IOP of $\geq 26 \mathrm{mmHg}$. All suitable patients presenting over a period of 1 year were enrolled in the current study.

Patients were excluded from this study if they demonstrated a history of ocular surgery/trauma, previous use of corticosteroids, contact lens use, dry eyes, corneal abnormality, or any condition that prevented reliable applanation tonometry. Also excluded were patients with systemic use of $\beta$-blockers, evidence of ocular infection, advanced cataract, inflammation, or history of renal or hepatic impairment. Also, patients were excluded who demonstrated a contraindication for topical use of $\beta$-blockers (bradycardia, decompensated heart failure, or reactive airway disease), with an allergy to sulpha, or a contraindication for the use of latanoprost.

\section{Procedures}

All patients signed an informed consent document before any procedures were performed. Patients then underwent an initial evaluation which included ocular medical history, Snellen visual acuity, Goldmann applanation tonometry, slit-lamp biomicroscopy, dilated funduscopy, and automated visual field testing
(Humphrey 24-2 visual field testing). Afterwards, qualified patients were randomly assigned to receive either latanoprost $0.005 \%$ (Pharmacia Hellas, Athens, Greece) once every evening (20:00) or timolol maleate/ dorzolamide fixed combination (Cosopt) twice daily (08:00 and 20:00) (Vianex S.A./MSD, Athens, Greece) for a period of 2 months before they were crossed over to the other treatment for the following 2 months. No washout period was allowed between treatment periods.

At months one and two in each treatment period, patients underwent Snellen visual acuity, slit-lamp biomicroscopy, and measurement of the IOP at the same time point (10:00 with a range of $+1 \mathrm{~h}$ ). Patients were dosed in the morning of each study visit (peak pressure evaluation for the timolol/dorzolamide fixed combination). All patients with baseline IOP values $>32 \mathrm{mmHg}$, or glaucomatous damage deemed severe, were seen earlier, at 1 and 2 weeks following the initiation of therapy and were followed up more frequently as needed according to the judgement of the investigator. When a patient was discontinued from a treatment period on account of side effects or inadequate IOP control, he was switched to the other treatment. These patients, however, were not included in the final efficacy analysis.

During the assessment of the IOP, the investigator who performed the pressure measurements was masked to the treatment regimen. The same calibrated instruments (Goldmann applanation tonometer) were used by the investigators at each site to measure the IOP. Patients were instructed regarding correct medication instillation and compliance. In this study all patients were advised and shown how to perform nasolacrimal occlusion for $1 \mathrm{~min}$ after instillation of the local medication. At each visit, nonsolicited ocular and systemic side effects that occurred during the treatment period were recorded. At the end of the study, patients were asked their preference (if any) for one of the medications and the reason for that preference.

Table 1 Ocular side effects

\begin{tabular}{lrrr}
\hline Side effect & $\begin{array}{c}\text { Timolol/dorzolamide } \\
\text { fixed }\end{array}$ & Latanoprost & P-value \\
\hline Conjunctival injection & 4 & 13 & 0.056 \\
Taste perversion & 14 & 0 & $<0.001$ \\
Burning on instillation & 12 & 3 & 0.036 \\
Foreign body sensation & 4 & 5 & $>0.99$ \\
Corneal SPK & 4 & 4 & 0.74 \\
Itchiness & 2 & 5 & 0.46 \\
Dry eye sensation & 1 & 3 & 0.66 \\
Watering & 1 & 0 & $>0.99$ \\
Blurring of vision & 1 & 0 & $>0.99$ \\
\hline
\end{tabular}

${ }^{\mathrm{a}} \mathrm{SPK}$ : superficial punctate epitheliopathy. 


\section{Statistics}

A $0.05 P$-value was used to declare significance. All tests were two-way analyses. The IOP was analysed by a paired Student's $t$-test. ${ }^{9}$ Visual acuity was analysed by a Wilcoxon signed-rank test and adverse events were analysed by McNemar test. ${ }^{9,10}$ In a patient with bilateral exfoliation glaucoma, one eye was randomly selected at the time of enrolment to be included in this study. Patient preference was analysed by a $\chi^{2}$ test. $^{10}$

\section{Results}

\section{Patients}

Out of the 65 patients enrolled, 54 completed both treatment periods. Of these 54 patients, 12 had exfoliation syndrome with ocular hypertension and 42 had exfoliation glaucoma. There were 21 females and 33 males. All patients were of Greek national origin and had an average age of $69.6 \pm 6.7$ years. The average visual acuity was $20 / 40$ Snellen. Of the 65 patients entered in this trial, 11 (16.9\%) were discontinued early from at least one period (nine periods for the fixed combination and eight for latanoprost) (Table 1).

\section{Intraocular pressure}

The baseline untreated IOP for all exfoliation patients was $31.2 \pm 6.5(29.5-33.0) \mathrm{mmHg}$ (mean \pm SD $(95 \%$ confidence intervals)). Both treatments significantly reduced the baseline IOP $(P<0.001)$ (Table 2$)$.

Latanoprost treatment reduced the IOP at 10:00 (14 h following dosing) following 1 month of chronic dosing to $19.0 \pm 3.7(18.0-20.1) \mathrm{mmHg}(-39.9 \%)$, and at 2 months to $18.9 \pm 4.1(17.8-20.0) \mathrm{mmHg}(-40.2 \%)$. The timolol/ dorzolamide fixed combination reduced the IOP at 10:00 ( $2 \mathrm{~h}$ following dosing) at 1 month to $17.8 \pm 3.0$ (17.0-18.6) $\mathrm{mmHg}(-43.7 \%)$ and at 2 months to $18.1 \pm 3.0(17.2-18.9)$ $\mathrm{mmHg}(-42.8 \%)$. The difference between the groups in these morning peak values was not statistically significant at 1 month $(P=0.06)$ or 2 months $(P=0.21)$. The fixed combination obtained a significantly lower mean maximum IOP in comparison with latanoprost $(P=0.04)$ (Table 2).

Eight patients were discontinued from at least one period because of inadequate IOP control. In six cases, both active treatment medicines failed to control the IOP of the patient. An additional two patients were discontinued from the latanoprost treatment period because of inadequate pressure control.

\section{Safety}

Table 1 shows the ocular side effects for both medications. There was a trend for a greater incidence of
Table 2 Intraocular pressure results (mean \pm SD (95\% confidence intervals))

\begin{tabular}{lcrc}
\hline Duration & $\begin{array}{c}\text { Timolol/dorzolamide } \\
\text { fixed combination }\end{array}$ & Latanoprost & P-value \\
\hline Baseline & $31.2 \pm 6.5$ & $31.2 \pm 6.5$ & 1.0 \\
& $(29.5-33.0)$ & $(29.5-33.0)$ & \\
Month 1 & $17.8 \pm 3.0$ & $19.0 \pm 3.7$ & 0.06 \\
& $(17.0-18.6)$ & $(18.0-20.1)$ & \\
Month 2 & $18.1 \pm 3.0$ & $18.9 \pm 4.1$ & 0.21 \\
& $(17.2-18.9)$ & $(17.8-20.0)$ & \\
Maximum & $18.6 \pm 3.1$ & $20.1 \pm 3.9$ & 0.04 \\
Minimum & $(17.8-19.5)$ & $(19.0-21.1)$ & \\
& $17.2 \pm 2.8$ & $17.9 \pm 3.5$ & 0.26 \\
Range & $(16.4-18.0)$ & $(16.9-18.9)$ & \\
& $-0.3 \pm 1.8$ & $0.1 \pm 2.9$ & 0.43 \\
Mean & $(-0.8-0.24)$ & $(-0.7-0.9)$ & \\
& $17.9 \pm 2.9$ & $19.0 \pm 3.6$ & 0.10 \\
& $(17.1-18.7)$ & $(18.0-20.0)$ & \\
\hline
\end{tabular}

conjunctival injection with latanoprost $(P=0.056)$, whereas the fixed combination had a greater incidence of taste perversion $(P<0.001)$ and burning on instillation $(P=0.036)$. Two patients were discontinued from the fixed combination owing to ocular intolerance.

A number of systemic side effects were recorded in the study. One patient on latanoprost had dizziness for several minutes following instillation. With the fixed combination treatment, there were two incidents of bradycardia, which did not lead to discontinuation. However, there were three new cases of shortness of breath with the fixed combination therapy. All three patients had no previous diagnosis of asthma. The symptoms resolved after discontinuation of the medication although one patient required hospitalization. One of these patients was also discontinued from treatment because of lack of IOP control.

\section{Patient preference}

Out of 54 patients (63\%) who completed the study, 34 preferred latanoprost treatment in this study. Of these, 23 patients preferred latanoprost because of greater convenience, 10 patients because of the lack of bitter taste or ocular burning as compared to the fixed combination, and one patient because vision was better with latanoprost.

In contrast, 11 patients $(20.3 \%)$ preferred the fixed combination treatment. Of these, as compared to latanoprost, four patients preferred the combination due to a lack of conjunctival hyperemia, two patients due to a lack of burning as compared to latanoprost, and one each because of lack of dizziness, dry eye, itching, and better tolerability. One patient also perceived the fixed 
combination to be easier to instill. The remaining nine patients $(16.7 \%)$ showed no preference between the two medications. There was a significant difference between treatment groups for preference in favour of latanoprost $(P<0.0001)$.

\section{Discussion}

Latanoprost was released commercially by Pharmacia, Inc. in 1996 as an ocular hypotensive agent. Latanoprost is an analogue of an $\mathrm{F}_{2} \alpha$ prostaglandin and is highly selective for the FP-receptor. ${ }^{11}$ Several studies have indicated that the mechanism of action of the IOP reduction by latanoprost is increased uveoscleral outflow. ${ }^{12-15}$ When compared to timolol maleate given twice daily, latanoprost $0.005 \%$ once daily has demonstrated either an equal or statistically greater reduction in IOP in regulatory trials (reduction of 6-8 $\mathrm{mmHg}(31-33 \%)) .{ }^{16-18}$ Side effects that have been described are iris colour darkening, eyelash growth, and conjunctival hyperaemia. ${ }^{19}$ A possible association with uveitis, recurrent corneal herpes keratitis, and cystoid macularoedema may also exist in some patients. ${ }^{20-22}$

A fixed combination of timolol maleate $0.5 \%$ and dorzolamide $2 \%$, Cosopt $^{\mathbb{R}}$ (Merck, Blue Bell, PA, USA), was released commercially in the US in $1998 .{ }^{23}$ The pharmacology of this product is related to its two active ingredients and it is prescribed for twice daily dosing. ${ }^{23}$ The regulatory trial by Boyle $e t$ al.$^{24}$ showed that the fixed combination reduced the IOP from untreated baseline by $7.7 \mathrm{mmHg}(27 \%)$. This trial, and another by Clineschmidt et $a{ }_{1}{ }^{25}$ demonstrated that the fixed-combination product further decreased the pressure 1.1-1.3 mmHg from timolol maleate at trough and $2.8 \mathrm{mmHg}$ at peak ( $2 \mathrm{~h}$ after dosing) ${ }^{25,26}$ Common side effects with the timolol maleate/dorzolamide fixed combination have been mostly related to the dorzolamide component, including bitter taste and stinging/burning on instillation.

The purpose of the present study was to evaluate the morning IOP control and safety of latanoprost given once every evening $v s$ the timolol maleate/dorzolamide fixed combination given twice daily in patients with newly diagnosed exfoliation glaucoma. We performed this trial in order to determine the efficacy of these two medicines in this important secondary glaucoma. We also evaluated these patients to determine if, because of higher baseline pressures, the differences between these two medicines would be exaggerated over those with primary openangle glaucoma. A limitation of the present study is that the design is effectively open-label, crossover with no interperiod washout. The relatively high untreated IOP level and the concomitant advanced damage seen in some of these patients would have made unethical the washout period.

This study found that both treatments statistically reduced the IOP from untreated baseline. At 1 month, there was a trend for greater reduction with the fixed combination $v s$ latanoprost, providing approximately a $1.2 \mathrm{mmHg}$ difference between groups $(P=0.06)$. At 2 months of therapy, this difference was $0.8 \mathrm{mmHg}$ but this difference was not significant $(P=0.21)$.

At both time points evaluated in the present study, the IOP reduction obtained (approximately 40\%) in previously untreated exfoliation patients was greater than that reported previously for primary open-angle glaucoma. ${ }^{3}$ It is not clear if this is due to the higher mean untreated baseline IOP, or whether newly diagnosed exfoliation glaucoma patients respond better to these medications. The current study supports the view that the treatment response in exfoliation glaucoma may vary from that seen in primary open-angle glaucoma. ${ }^{1}$ The greater initial reduction of IOP with latanoprost and the fixed combination in exfoliation glaucoma at 2 months may decline with chronic treatment. A better initial hypotensive response to surgery has also been reported in exfoliation glaucoma vs primary open-angle glaucoma. ${ }^{1,8}$

In the current study, our findings were consistent with our previous research that at morning peak the fixed combination showed a trend to better efficacy than latanoprost. ${ }^{4}$ The difference between groups was slightly greater in the exfoliation patients $(0.8 \mathrm{mmHg})$ in the current study than in primary open-angle glaucoma patients $(0.3 \mathrm{mmHg})$ at the 10:00 time point shown previously. ${ }^{4}$ These results could raise the question if in the evening, consistent with the previous $24 \mathrm{~h}$ study, ${ }^{4}$ the fixed combination might provide better pressure control than latanoprost in exfoliation glaucoma patients.

A higher number of patients (16.9\%) were not controlled in this study with these formulations than usually has been observed in comparative trials. This was probably because the patients had exfoliation glaucoma, and their baseline pressures were higher and thus less likely to be controlled by a single medication. Six patients failed to be controlled in both periods by each medication, and two additional patients were not controlled on latanoprost.

Although latanoprost showed a trend of not reducing the IOP as well as the fixed combination at 10:00 in this study, it did show at least three potential advantages in these newly treated exfoliation patients. First, despite a higher incidence of conjunctival hyperaemia, patient preference was generally given to latanoprost because of its once daily dosing, and lack of taste perversion as well as stinging with the fixed combination. This finding may indicate that latanoprost could increase compliance in 
newly diagnosed patients. Second, three of the newly treated patients with the fixed combination had new onset asthma probably related to the systemic $\beta$ blockade. One of these patients was hospitalized. This again shows the need for care in prescribing $\beta$-blockers, especially in previously untreated elderly patients, and for physicians to be fully aware of the potential of severe systemic side effects. ${ }^{27,28}$ Third, latanoprost is a single active agent, whereas the fixed combination contains two active agents necessitating physician awareness of the efficacy and safety profile of both these agents when prescribing.

To date, there are few comparative studies evaluating the efficacy of the new medications in exfoliation glaucoma. ${ }^{29-31}$ It is generally thought that exfoliation patients are more difficult to control with medical therapy than those with primary open-angle glaucoma. ${ }^{1,2,8}$ These observations, however, do not take into account the impact of the newer medications (latanoprost, the fixed combination, etc) on the success of medical therapy in exfoliation glaucoma. Further studies are required to address this issue.

The present study suggests that both latanoprost and the timolol/dorzolamide fixed combination are efficacious in reducing the IOP in exfoliation glaucoma. However, the long-term efficacy of latanoprost and the fixed combination in exfoliation glaucoma requires further study. This trial did not evaluate the IOP at the morning trough level for the fixed combination, or the evening trough level for latanoprost. This would have required a complete 24 -h diurnal curve with these medications. The efficacy of these medicines could have differed at other time points. In addition, this study did not evaluate patients previously treated with medications. Previous treatment could have changed their efficacy, or patients' perception of the side effects from these medications.

\section{References}

1 Ritch R, Schlötzer-Schrehardt U. Exfoliation syndrome. Surv Ophthalmol 2001; 45: 265-315.

2 Vesti E, Kivela T. Exfoliation syndrome and exfoliation glaucoma. Prog Retin Eye Res 2000; 19: 345-368.

3 Fechtner R, Clineschmidt C, Getson A, Adamsons I. A three month, parallel, randomized, observer-masked, multicenter study comparing Cosopt ${ }^{\mathrm{TM}}$ to Xalatan ${ }^{\mathrm{TM}}$ in patients with elevated IOP. Invest Ophthalmol Vis Sci 1999; 40: S665.

4 Konstas AGP, Papapanos P, Tersis I, Houliara D, Holmes KT, Stewart WC. A comparison of the efficacy and safety of latanoprost $0.005 \%$ every evening versus timolol/ dorzolamide fixed combination twice daily. Invest Ophthalmol Vis Sci 2001; 42: S420.

5 Konstas AGP, Maltezos AC, Gandi S, Hudgins AC, Stewart WC. Comparison of the 24 hour intraocular pressure reduction with two dosing regimes of latanoprost and timolol in patients with open-angle glaucoma. Am J Ophthalmol 1999; 128: 15-20.

6 Konstas AGP, Mantziris DA, Stewart WC. Diurnal intraocular pressure in untreated exfoliation and primary open-angle glaucoma. Arch Ophthalmol 1997; 115: 182-185.

7 Vogel R. The impact of entry criteria on the baseline IOP in glaucoma studies: a meta-analysis. Invest Ophthalmol Vis Sci 1996; 37: S1099.

8 Konstas AGP, Jay JL, Marshall GE, Lee WR. Prevalence, diagnostic features, and response to trabeculectomy in exfoliation glaucoma. Ophthalmology 1993; 100: 619-627.

9 Book SA. Essentials of Statistics. McGraw-Hill: New York, 1978.

10 Siegel S. Non Parametric Statistics. Series in Psychology. McGraw-Hill: New York, 1956.

11 Stjernschantz JW. From PGF(2alpha)-isopropyl ester to latanoprost: a review of the development of xalatan: the Proctor Lecture. Invest Ophthalmol Vis Sci 2001; 42: 1134-1145.

12 Nilsson SFE, Samuelsson M, Bill A, Stjernschantz J. Increased uveoscleral outflow as a possible mechanism of ocular hypotension caused by prostaglandin $\mathrm{F}_{2 \mathrm{a}}-1$ isopropylester in the cynomolgus monkey. Exp Eye Res 1989; 48: 707-716.

13 Gabelt BT, Kaufman PL. Prostaglandin $\mathrm{F}_{2 \mathrm{a}}$ increases uveoscleral outflow in the cynomolgus monkey. Exp Eye Res 1989; 49: 389-402.

14 Kerstetter JR, Brubaker RF, Wilson SE, Kullerstrand LJ Prostaglandin $\mathrm{F}_{2 \mathrm{a}}$-1-isopropylester lowers intraocular pressure without decreasing aqueous humor flow. Am J Ophthalmol 1988; 105: 30-34.

15 Toris CB, Camras CD, Yablonski ME. Effects of PhXA41, a new prostaglandin $\mathrm{F}_{2 \mathrm{a}}$ analog, on aqueous humor dynamics in human eyes. Ophthalmology 1993; 100: 1297-1304.

16 Alm A, Stjernschantz J, Scandinavian Latanoprost Study Group. Effects on intraocular pressure and side effects of $0.005 \%$ latanoprost applied once daily, evening or morning: a comparison with timolol. Ophthalmology 1995; 102: 1743-1752.

17 Camras CB, United States Latanoprost Study Group. Comparison of latanoprost and timolol in patients with ocular hypertension and glaucoma: a six-month, masked, multicenter trial in the United States. Ophthalmology 1996; 103: 138-147.

18 Watson P, Stjernschantz J, Scandinavian Latanoprost Study Group. A six-month, randomized, double-masked study comparing latanoprost with timolol in open-angle glaucoma and ocular hypertension. Ophthalmology 1996; 103: 126-137.

19 Stewart WC. Current and Future Glaucoma Therapy, Ciba Vision Ophthalmics: Duluth, GA, 1997, pp 1-43.

20 Smith SL, Pruitt CA, Sine CS, Hudgins AC, Stewart WC. Latanoprost $0.005 \%$ and anterior segment uveitis. Acta Ophthalmol Scand 1999; 77: 668-672.

21 Moroi SE, Gottfredsdottir MS, Schteingart MT, Elner SG, Lee CM, Schertzer RM et al. Cystoid macular edema associated with latanoprost therapy in a case series of patients with glaucoma and ocular hypertension. Ophthalmology 1999; 106: 1024-1029.

22 Kaufman HE, Varnell ED, Thompson HW. Latanoprost increases the severity and recurrence of herpetic keratitis in the rabbit. Am J Ophthalmol 1999; 127: 531-536.

23 Stewart WC. New perspectives in the medical treatment of glaucoma. Curr Opin Ophthalmol 1999; 127: 142-147. 
24 Boyle JE, Ghosh K, Gieser DK, Adamsons IA, DorzolamideTimolol Study Group. A randomized trial comparing the dorzolamide-timolol combination given twice daily to monotherapy with timolol and dorzolamide. Ophthalmology 1998; 105: 145-151.

25 Clineshmidt CM, Williams RD, Snyder E, Adamsons IA. A randomized trial in patients inadequately controlled on timolol alone comparing the dorzolamide-timolol combination to monotherapy with timolol or dorzolamide. Ophthalmology 1999; 106: 17-24.

26 Boyle JE, Ghosh K, Gieser DK, Adamsons IA. A randomized trial comparing the dorzolamide-timolol combination given twice daily to monotherapy with timolol or dorzolamide. Ophthalmology 1999; 106: 10-16.

27 Hutzelmann J, Owens S, Sedden A, Adamsons I, Vargas E. Comparison of the safety and efficacy of the fixed combination of dorzolamide/timolol and the concomitant administration of dorzolamide and timolol: a clinical equivalence study. International Clinical Equivalence Study Group. Br J Ophthalmol 1998; 82: 1249-1253.

28 Stewart WC, Garrison PM. $\beta$-blocker-induced complications and the glaucoma patient: newer treatments to help reduce systemic side effects. Arch Int Med 1998; 158: 221-226.

29 Heijl A, Strahlman E, Sverrisson T et al. A comparison of dorzolamide and timolol in patients with pseudoexfoliation and glaucoma or ocular hypertension. Ophthalmology 1997; 104: 137-142.

30 Konstas AGP, Maltezos T, Bufidis T, Hudgins AC, Stewart WC. 24 hour control of IOP with dorzolamide and timolol maleate in exfoliation and primary open-angle glaucoma. Eye 2000; 14: 73-77.

31 Konstas AGP, Lake S, Maltezos AC, Holmes KT, Stewart WC. 24 hour intraocular pressure reduction with latanoprost compared to pilocarpine as third line therapy in exfoliation glaucoma. Eye 2001; 15: 59-62. 